\title{
The Detector Efficiency Question with EDS
}

Frank Eggert ${ }^{1}$, Jens Rafaelsen ${ }^{1}$, Felix Reinauer ${ }^{1}$, Patrick Camus ${ }^{1}$ and Ulrich Gernert ${ }^{2}$

${ }^{1}$ Ametek / EDAX LLC, Mahwah, New Jersey, United States, ${ }^{2}$ Technische Universität Berlin, Center f. El. Micr. (ZELMI), Berlin, Berlin, Germany

The knowledge about EDS detector efficiency is a core question with standardless quantification of measured spectra, to convert the measured X-ray counts into the values which were really emitted from specimen. Quantmodels for sample composition calculations always start with the input of emitted X-rays, not with the measured X-rays. The detector influence cancels out if measured P/B are used or when net-counts of a known standard are measured with the same detector. But even if the standards were measured with another detector (or even with another type) it matters much to know the real efficiency with each detector.

The major part of the efficiency property is dominated by the atmospheric window. Therefore, it was tried previously to measure this at Synchrotron facilities [1], e.g. in white beam. Here it is possible to calculate the incoming X-ray energy distribution from fundamental parameters and physics. The cited work has investigated a sandwich window composed of $\mathrm{SiO} 2$ and $\mathrm{Si} 3 \mathrm{~N} 4$ with about $0.8 \mu \mathrm{m}$ thickness in total with no support grid. While the window itself affects the low energy part of the efficiency curve the most, it is the mechanical support grid which can influence higher energies. The total detector efficiency can vary much due to different designs [2]. The newly introduced Si3N4 window [3,4] is only $0.04 \mu \mathrm{m}$ thick and equipped with a $15 \mu \mathrm{m} \mathrm{Si}$ support grid. The grid is already transparent below $10 \mathrm{keV}$. The analytical software must consider the active role of this grid between 1 and $10 \mathrm{keV}$. This was assumed not to be required for the traditional Moxtek window [5-6] with $380 \mu \mathrm{m}$ grid thickness.

Both windows are known with their specification and they were measured at Synchrotron facilities [4-6] and characterized for use with EDS in SEM or TEM. Nevertheless, we have found discrepancies which were first not possible to explain. The new Si3N4 window detectors are with measured low-energy X-rays efficiencies much higher than the expectation is based on theoretical efficiency curves which are in alignment with the measured and published Synchrotron measured transmission curves. The deviations were visible in quantitative results (e.g. too high measured Oxygen), similar in direct net-count-ratios of low energy element lines vs. higher energies comparison between new window detectors vs. old. That means that the new window is even significantly better than published (Fig.1), and in the reality thinner but from an unknown mechanism?

Then we have started a comparison measurement between the detector types parallel in one SEM, in 'absolute' controlled measurement strategy. There were also found several discrepancies in the higher energy region. Until this point, all thoughts were based on assuming the known and published transmission curves were proper in an SEM configuration. But this is obviously not true. It is a fallacy to estimate the Moxtek lamellas do not affect the energy dependent detector efficiencies. The difference of the normal SEM/EDS configuration and the Synchrotron radiation measurements is that the SEM detector is very close to the point-source. And therefore, the SEM/EDS is with a strong divergent X-ray (in contrast to Synchrotron parallel beam). It was already mentioned a shadow effect in [6]: "When the user places his detector at a short distance from the specimen to get a large solid angle for detection, the radiation emitted nonparallel to the lamellas of the window grid will be partially absorbed." We have then assumed that the Si3N4 window with the thin grid has no extra divergency effects for normal SEM/EDS situation, and therefore this curve was used as the new reference in SEM. Our investigations have then shown that it is required to re-evaluate the old used assumptions with old window EDS detector efficiencies (Fig.2). The lamellas have divergence effects with energy dependent partial 
X-ray transmission, inclined low-energy X-rays cannot pass the grid edges, whereas higher energies are able. One can describe it alternatively as the solid angle of the detector gets bigger with higher energies. This has in other view the effect that the low energy relative detector-efficiency is lower than assumed in ratio to $\mathrm{Cu}-\mathrm{Ka}$, as an example. The divergence effect by grid-lamellas can be a matter of variation by detector size and detector distance from sample, not with new Si3N4 window.

\section{Efficiency of $\mathrm{Si}_{3} \mathrm{~N}_{4}$ window detector}

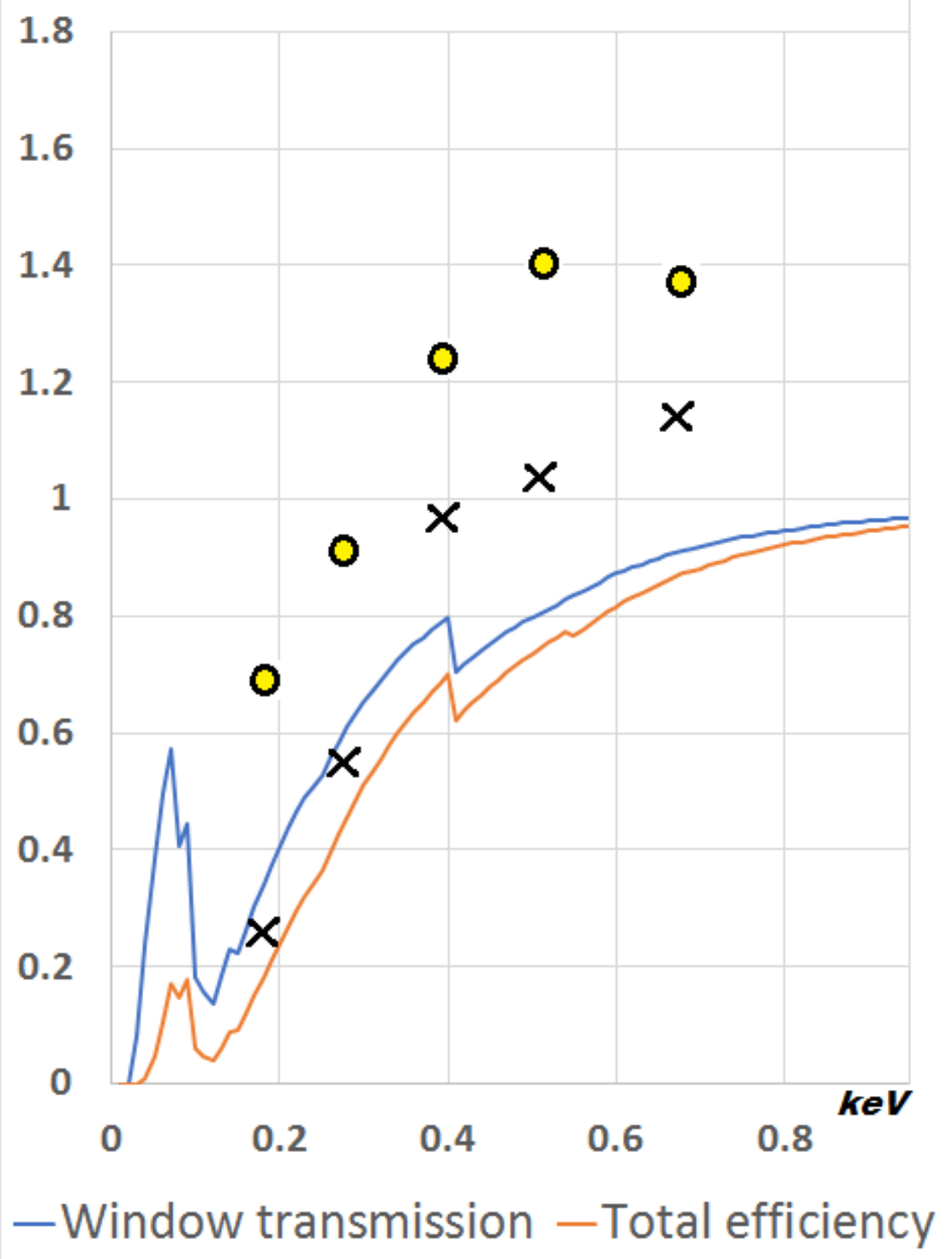

Figure 1. Theory efficiency curves of a new EDAX detector with Si3N4 window. The bullets are one early example for measured efficiencies in relation to higher energy elements (binary samples), based on assumption the known old window detector efficiency is the truth. The crosses are the most recent results with parallel detector setup with both assumed clean windows. It has raised questions because it is much better in comparison to old detectors than assumed. 


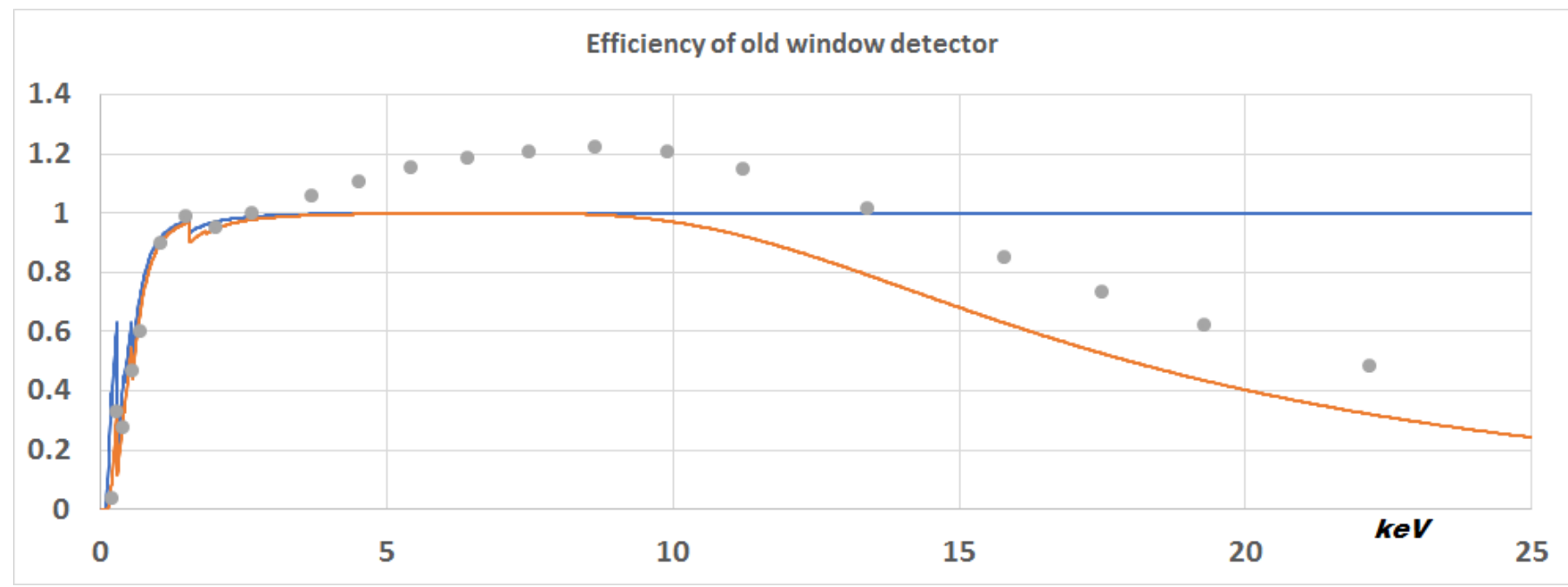

Figure 2. Efficiency curve with an old EDAX detector window. The bullets are now the corrected detector total efficiency of old window if assumed the new Si3N4 efficiency curve is also proper in SEM. It considers the lamella normal transmission about energies $>10 \mathrm{keV}$ and additional the new assumed divergence effect. With energies $<1 \mathrm{keV}$ the efficiency is then almost like in parallel beam (if the window is not dirty).

\section{References}

[1] F Eggert, M Maneck, M Krumrey, F Scholze, E Tegeler, Rev. of Sc. Instrum. 62 (1991) 741

[2] P P Camus, Microsc. Microanal. 23 (2017) 1064

[3] EDAX Technical Note (2014)

[4] P T Törmä at al. IEEE Transact. On Nuclear Science 61 (2014) 695

[5] F Scholze, M Procop, X-Ray Spectrom. 34 (2005) 473

[6] M Alvisi at al, Microsc. Microanal. 12 (2006) 406 